\title{
On infinitesimal conformal transformations with respect to the Cheeger-Gromoll metric
}

\author{
Aydin Gezer, Lokman Bilen
}

\begin{abstract}
The present paper deals with the classification of infinitesimal fibrepreserving conformal transformations on the tangent bundle, equipped with the Cheeger-Gromoll metric.
\end{abstract}

\section{Introduction}

Let $M$ be an $n$-dimensional manifold and $T M$ its tangent bundle. We denote by $\Im_{s}^{r}(M)$ the set of all tensor fields of type $(r, s)$ on $M$. Similarly, we denote by $\Im_{s}^{r}(T M)$ the corresponding set on $T M$. We also note that in the present paper everything will be always discussed in the $C^{\infty}$-category, and manifolds will be assumed to be connected and of dimension $n>1$.

Let $M$ be a Riemannian manifold with a Riemannian metric $g$ and $X$ be a vector field on $M$. Let us consider the local one-parameter group $\left\{\phi_{t}\right\}$ of local transformations of $M$ generated by $X$. The vector field $X$ is called an infinitesimal conformal transformation if each $\phi_{t}$ is a local conformal transformation of $M$. As is well known, the vector field $X$ is an infinitesimal conformal transformation or conformal vector field on $M$ if and only if there exist a scalar function $\rho$ on $M$ satisfying $L_{X} g=2 \rho g$, where $L_{X}$ denotes the Lie derivation with respect to $X$. Especially, the vector field $X$ is called an infinitesimal homothetic one when $\rho$ is constant.

Let $T M$ be the tangent bundle over $M$ and $\Phi$ be a transformation of $T M$. If the transformation $\Phi$ preserves the fibres, it is called a fibre-preserving

Key Words: Cheeger-Gromoll metric, fibre-preserving vector field, infinitesimal conformal transformation.

2010 Mathematics Subject Classification: 53B21, 53A45.

Received: January, 2011.

Accepted: February, 2012. 
transformation. Consider a vector field $\tilde{X}$ on $T M$ and the local one-parameter group $\left\{\Phi_{t}\right\}$ of local transformations of $T M$ generated by $\tilde{X}$. The vector field $\tilde{X}$ is called an infinitesimal fibre-preserving transformation if each $\Phi_{t}$ is a local fibre-preserving transformation of $T M$. An infinitesimal fibre-preserving transformation $\tilde{X}$ on $T M$ is called an infinitesimal fibre-preserving conformal transformation if each $\Phi_{t}$ is a local fibre-preserving conformal transformation of $T M$. Let $\tilde{g}$ be a Riemannian or pseudo-Riemannian metric on $T M$. $\tilde{X}$ is an infinitesimal conformal transformation of $T M$ if and only if there exist a scalar function $\Omega$ on $T M$ such that $L_{\tilde{X}} \tilde{g}=2 \Omega \tilde{g}$, where $L_{\tilde{X}}$ denotes the Lie derivation with respect to $\tilde{X}$. An infinitesimal conformal transformation $\tilde{X}$ is called essential if $\Omega$ depends only on $\left(y^{i}\right)$ with respect to the induced coordinates $\left(x^{i}, y^{i}\right)$ on $T M$, and is called inessential if $\Omega$ depends only $\left(x^{i}\right)$, that is, $\Omega$ is a constant on each fibre of $T M$. In this case, $\Omega$ induces a function on $M$.

The geometry of tangent bundles goes back to the fundamental paper [27] of Sasaki published in 1958. He uses a given Riemannian metric $g$ on a differentiable manifold $M$ to construct a metric $\tilde{g}$ on the tangent bundle $T M$ of $M$. Today this metric is a standard notion in the differential geometry called the Sasaki metric (or the metric I+III). For a given Riemannian metric $g$ on a differentiable manifold $M$, there are well known Riemannian or pseudoRiemannian metrics on $T M$, constructed from the metric $g$, as follows:

1. The complete lift metric or the metric $I I$

2. The metric $I+I I$

3. The Sasaki metric or the metric $I+I I I$

4. The metric $I I+I I I$

where $I=g_{i j} d x^{i} d x^{j}, I I=2 g_{i j} d x^{i} \delta y^{j}, I I I=g_{i j} \delta y^{i} \delta y^{j}$ are all quadratic differential forms defined globally on the tangent bundle $T M$ over $M$ (for details, see [[33], p.137-177]). Yamauchi [30] proved that every infinitesimal fibre-preserving conformal transformation on $T M$ with the metric $I+I I I$ is homothetic and it induces an infinitesimal homothetic transformation on $M$. Also, in the case when $M$ is a complete, simply connected Riemannian manifold with a Riemannian metric, Hasegawa and Yamauchi [11] showed that the Riemannian manifold $M$ is isometric to the standard sphere when the tangent bundle $T M$ equipped with the metric $I+I I$ admits an essential infinitesimal conformal transformation. In [9], the first author has studied the similar problem in $[30,31]$ with respect to the synectic lift metric on the tangent bundle.

All the preceding metrics belong to the wide class of the so-called $g$-natural metrics on the tangent bundle, initially classified by Kowalski and Sekizawa 
[13] and fully characterized by Abbassi and Sarih [1, 2, 3, 4] (see also [12, 6] for other presentation of the basic result from [13] and for more details about the concept of naturality). Another well-known $g$-natural Riemannian metric $g_{C G}$ had been defined, some years before, by Muso and Tricerri [15] who, inspired by the paper [7] of Cheeger and Gromoll, called it the Cheeger-Gromoll metric. The metric was defined by Cheeger and Gromoll; yet, there were Musso and Tricerri who wrote down its expression, constructed it in a more "comprehensible" way, and gave it the name. The Levi-Civita connection of $g_{C G}$ and its Riemannian curvature tensor are calculated by Sekizawa in [28] (for more details see [10]). In [4], Abbassi and Sarih classified Killing vector fields on $\left(T M, g_{C G}\right)$; that is, they found general forms of all Killing vector fields on $\left(T M, g_{C G}\right)$. Also, they showed that if $\left(T M, g_{C G}\right)$ is the tangent bundle with the Cheeger-Gromoll metric $g_{C G}$ of a Riemannian, compact and orientable manifold $(M, g)$ with vanishing first and second Betti numbers, then the Lie algebras of Killing vector fields on $(M, g)$ and on $\left(T M, g_{C G}\right)$ are isomorphic. Finally, they showed that the sectional curvature of the tangent bundle $\left(T M, g_{C G}\right)$ with the Cheeger-Gromoll metric $g_{C G}$ of a Riemannian manifold $(M, g)$ is never constant. In [26], Salimov and Kazimova investigated geodesics on the tangent bundle with respect to the Cheeger-Gromoll metric $g_{C G}$. Different types of metrics on the tangent bundle of a Riemannian manifold were also studied in $[5,14,16,17,18,19,20,21,22,23,24,25]$

The purpose of the present paper is to characterize infinitesimal fibrepreserving conformal transformations with respect to the Cheeger-Gromoll metric $g_{C G}$ on the tangent bundle $T M$ of a Riemannian manifold $M$. In Theorem 3.2 , we give a necessary and sufficient condition for the vector field $\tilde{X}$ on the tangent bundle with the Cheeger-Gromoll metric $g_{C G}$ to be an infinitesimal fibre-preserving conformal transformation. This condition is represented by a set of relations involving certain tensor fields on $M$ of type $(1,0)$ and $(1,1)$. We obtain these relations by giving the formula $L_{\tilde{X}} g_{C G}=2 \Omega g_{C G}$ in an adapted frame. The paper ends two Corollaries which follow immediately from Theorem 3.2 and its Proof.

\section{Preliminaries}

\subsection{Cheeger-Gromoll metric on the tangent bundle}

Let $T M$ be the tangent bundle over an $n$-dimensional manifold $M$, and $\pi$ the natural projection $\pi: T M \rightarrow M$. Let the manifold $M$ be covered by a system of coordinate neighborhoods $\left(U, x^{i}\right)$, where $\left(x^{i}\right), i=1, \ldots, n$ is a local coordinate system defined in the neighborhood $U$. Let $\left(y^{i}\right)$ be the Cartesian coordinates in each tangent space $T_{P} M$ at $P \in M$ with respect to the natural 
base $\left\{\left.\frac{\partial}{\partial x^{i}}\right|_{P}\right\}, P$ being an arbitrary point in $U$ whose coordinates are $\left(x^{i}\right)$. Then we can introduce local coordinates $\left(x^{i}, y^{i}\right)$ on open set $\pi^{-1}(U) \subset T M$. We call them induced coordinates on $\pi^{-1}(U)$ from $\left(U, x^{i}\right)$. The projection $\pi$ is represented by $\left(x^{i}, y^{i}\right) \rightarrow\left(x^{i}\right)$. We use the notions $x^{I}=\left(x^{i}, x^{\bar{i}}\right)$ and $x^{\bar{i}}=y^{i}$. The indices $i, j, \ldots$ run from 1 to $n$, the indices $\bar{i}, \bar{j}, \ldots$ run from $n+1$ to $2 n$. Summation over repeated indices is always implied.

Let $X=X^{i} \frac{\partial}{\partial x^{i}}$ be the local expression in $U$ of a vector field $X$ on $M$. Then the vertical lift ${ }^{V} X$, the horizontal lift ${ }^{H} X$ and the complete lift ${ }^{C} X$ of $X$ are given, with respect to the induced coordinates, by

$$
\begin{gathered}
{ }^{V} X=X^{i} \partial_{\bar{i}}, \\
{ }^{H} X=X^{i} \partial_{i}-y^{s} \Gamma_{s k}^{i} X^{k} \partial_{\bar{i}},
\end{gathered}
$$

and

$$
{ }^{C} X=X^{i} \partial_{i}+y^{s} \partial_{s} X^{i} \partial_{\bar{i}}
$$

where $\partial_{i}=\frac{\partial}{\partial x^{i}}, \partial_{\bar{i}}=\frac{\partial}{\partial y^{i}}$ and $\Gamma_{s k}^{i}$ are the coefficients of the Levi-Civita connection $\nabla$ of $g$.

Suppose that we are given a tensor field $S \in \Im_{q}^{p}(M), q>1$, on $M$. We define a tensor field $\gamma S \in \Im_{q-1}^{p}(T M)$ on $\pi^{-1}(U)$ by

$$
\gamma S=\left(y^{e} S_{e i_{2} \ldots i_{q}}^{j_{1} \ldots j_{p}}\right) \partial_{\bar{j}_{1}} \otimes \ldots \otimes \partial_{\bar{j}_{p}} \otimes d x^{i_{2}} \otimes \ldots \otimes d x^{i_{q}}
$$

with respect to the induced coordinates $\left(x^{i}, y^{i}\right)([33]$, p.12). The tensor field $\gamma S$ defined on each $\pi^{-1}(U)$ determines a global tensor field on $T M$. We easily see that $\gamma A$ has components, with respect to the induced coordinates $\left(x^{i}, y^{i}\right)$,

$$
(\gamma A)=\left(\begin{array}{c}
0 \\
y^{i} A_{i}^{j}
\end{array}\right)
$$

for any $A \in \Im_{1}^{1}(M)$ and $(\gamma A)\left({ }^{V} f\right)=0, f \in \Im_{0}^{0}(M)$, i.e. $\gamma A$ is a vertical vector field on $T M$.

Explicit expression for the Lie bracket [,] of the tangent bundle $T M$ is given by Dombrowski [8]. The bracket products of vertical and horizontal vector fields are given by the formulas:

$$
\begin{aligned}
& {\left[{ }^{H} X,{ }^{H} Y\right]={ }^{H}[X, Y]-\gamma(R(X, Y))} \\
& {\left[{ }^{H} X,{ }^{V} Y\right]={ }^{V}\left(\nabla_{X} Y\right)} \\
& {\left[{ }^{V} X,{ }^{V} Y\right]=0}
\end{aligned}
$$

for all vector fields $X$ and $Y$ on $M$, where $R$ is the Riemannian curvature of $g$ defined by $R(X, Y)=\left[\nabla_{X}, \nabla_{Y}\right]-\nabla_{[X, Y]}$ and $\gamma(R(X, Y))$ is a tensor field of 
type $(1,0)$ on $T M$, which is locally expressed as $\gamma(R(X, Y))=y^{s} R_{j k s}^{i} X^{j} Y^{k} \partial_{i}$ with respect to the induced coordinates.

Let us consider a vector field $X=X^{i} \partial_{i}$ and the corresponding covector field $g_{X}=g_{i j} X^{i} d x^{j}$ on $U$. Then $\gamma g_{X} \in \Im_{0}^{0}(M)$ is a function on $\pi^{-1}(U)$ defined by $\gamma g_{X}=y^{i} g_{i j} X^{j}$ with respect to the induced coordinates $\left(x^{i}, y^{i}\right)$. Now, denote by $r$ the norm a vector $y=\left(y^{i}\right)$, i.e. $r^{2}=g_{j i} y^{j} y^{i}$. The CheegerGromoll metric $g_{C G}$ on the tangent bundle $T M$ is given by

$$
\begin{aligned}
& g_{C G}\left({ }^{H} X,{ }^{H} Y\right)={ }^{V}(g(X, Y)), \\
& g_{C G}\left({ }^{H} X,{ }^{V} Y\right)=0, \\
& g_{C G}\left({ }^{V} X,{ }^{V} Y\right)=\frac{1}{1+r^{2}}\left[{ }^{V}(g(X, Y))+\left(\gamma g_{X}\right)\left(\gamma g_{Y}\right)\right],
\end{aligned}
$$

for all $X, Y \in \Im_{0}^{1}(M)$, where ${ }^{V}(g(X, Y))=(g(X, Y)) \circ \pi$.

\subsection{Basic formulas in adapted frames}

With a torsion-free affine connection $\nabla$ given on $M$, we can introduce on each induced coordinate neighborhood $\pi^{-1}(U)$ of $T M$ a frame field which is very useful in our computation. In each local chart $U \subset M$, we put $X_{(j)}=$ $\frac{\partial}{\partial x^{j}}, j=1, \ldots, n$. Then from $(2.1)$ and $(2.2)$, we see that these vector fields have, respectively, local expressions

$$
\begin{gathered}
{ }^{H} X_{(j)}=\delta_{j}^{h} \partial_{h}+\left(-y^{s} \Gamma_{s j}^{h}\right) \partial_{\bar{h}} \\
{ }^{V} X_{(j)}=\delta_{j}^{h} \partial_{\bar{h}}
\end{gathered}
$$

with respect to the natural frame $\left\{\partial_{h}, \partial_{\bar{h}}\right\}$, where $\delta_{j}^{h}$-Kronecker delta. These $2 n$ vector fields are linear independent and generate, respectively, the horizontal distribution of $\nabla$ and the vertical distribution of $T M$. We have call the set $\left\{{ }^{H} X_{(j)},{ }^{V} X_{(j)}\right\}$ the frame adapted to the affine connection $\nabla$ in $\pi^{-1}(U) \subset$ $T M$. On putting

$$
\begin{aligned}
& E_{j}={ }^{H} X_{(j)}, \\
& E_{\bar{j}}={ }^{V} X_{(j)},
\end{aligned}
$$

we write the adapted frame as $\left\{E_{\lambda}\right\}=\left\{E_{j}, E_{\bar{j}}\right\} .\left\{d x^{h}, \delta y^{h}\right\}$ is the dual frame of $\left\{E_{i}, E_{\bar{i}}\right\}$, where $\delta y^{h}=d y^{h}+y^{b} \Gamma_{b a}^{h} d x^{a}$. By the straightforward calculation, we have the following:

2.3 Lemma. The Lie brackets of the adapted frame of TM satisfy the following identities:

$$
\left\{\begin{array}{l}
{\left[E_{j}, E_{i}\right]=y^{b} R_{i j b}^{a} E_{\bar{a}}} \\
{\left[E_{j}, E_{\bar{i}}\right]=\Gamma_{j i}^{a} E_{\bar{a}}} \\
{\left[E_{\bar{j}}, E_{\bar{i}}\right]=0}
\end{array}\right.
$$


where $R_{i j b}^{a}$ denote the components of the curvature tensor of $M$ [31].

Using (2.1), (2.2) and (2.3), we have

$$
\begin{aligned}
{ }^{H} X & =\left(\begin{array}{c}
X^{j} \delta_{j}^{h} \\
-X^{j} \Gamma_{s j}^{h} y^{s}
\end{array}\right)=X^{j}\left(\begin{array}{l}
\delta_{j}^{h} \\
-\Gamma_{s j}^{h} y^{s}
\end{array}\right)=X^{j} E_{j} \\
{ }^{V} X & =\left(\begin{array}{l}
0 \\
X^{h}
\end{array}\right)=\left(\begin{array}{l}
0 \\
X^{j} \delta_{j}^{h}
\end{array}\right)=X^{j}\left(\begin{array}{l}
0 \\
\delta_{j}^{h}
\end{array}\right)=X^{j} E_{\bar{j}},
\end{aligned}
$$

and

$$
\begin{aligned}
{ }^{C} X & =\left(\begin{array}{c}
X^{j} \delta_{j}^{h} \\
y^{s} \partial_{s} X^{j}
\end{array}\right) \\
& =X^{j}\left(\begin{array}{l}
\delta_{j}^{h} \\
-\Gamma_{s j}^{h} y^{s}
\end{array}\right)+y^{m} \nabla_{m} X^{j}\left(\begin{array}{l}
0 \\
\delta_{j}^{h}
\end{array}\right)=X^{j} E_{j}+y^{m} \nabla_{m} X^{j} E_{\bar{j}}
\end{aligned}
$$

with respect to the adapted frame $\left\{E_{\lambda}\right\}$.

We shall need a new lift of vector fields on $M$. For any vector field $Y \in$ $\Im_{0}^{1}(M)$ with the components $\left(Y^{h}\right),{ }^{V^{\prime}} Y$ is a vector field on $T M$ defined by

$$
V^{\prime} Y=\left\{\left(1-r^{2}\right) Y^{a}+g_{k r} Y^{k} y^{r} y^{a}\right\} E_{\bar{a}},
$$

with respect to the adapted frame $\left\{E_{\lambda}\right\}$. Clearly the lift ${ }^{\prime} Y$ is a smooth vector field on $T M$. Remark that ${ }^{\prime} Y$ is a vertical vector field on $T M$. In fact, $f \in \Im_{0}^{0}(M) ; V^{\prime} Y V(f)=0$.

Let $\tilde{X}$ be a vector field on $T M$ with components $\left(v^{h}, v^{\bar{h}}\right)$ with respect to the adapted frame $\left\{E_{h}, E_{\bar{h}}\right\}$. Then $\tilde{X}$ is a fibre-preserving vector field on $T M$ if and only if $v^{h}$ depend only on the variables $\left(x^{h}\right)$. Therefore, every fibrepreserving vector field $\tilde{X}$ on $T M$ induces a vector field $X=v^{h} \frac{\partial}{\partial x^{h}}$ on $M$. Also, it is well-known that ${ }^{C} X,{ }^{V} X,{ }^{V^{\prime}} X$ and ${ }^{H} X$ are fibre-preserving vector fields on $T M$.

Let $L_{\tilde{X}}$ be the Lie derivation with respect to the fibre-preserving vector field $\tilde{X}$, then we have the following Lemma:

2.4 Lemma. (see [30, 31]) The Lie derivations of the adapted frame and its dual basis with respect to $\tilde{X}=v^{h} E_{h}+v^{\bar{h}} E_{\bar{h}}$ are given as follows:

(1) $L_{\tilde{X}} E_{h}=-\partial_{h} v^{a} E_{a}+\left\{y^{b} v^{c} R_{h c b}^{a}-v^{\bar{b}} \Gamma_{b h}^{a}-E_{h}\left(v^{\bar{a}}\right)\right\} E_{\bar{a}}$

(2) $L_{\tilde{X}} E_{\bar{h}}=\left\{v^{b} \Gamma_{b h}^{a}-E_{\bar{h}}\left(v^{\bar{a}}\right)\right\} E_{\bar{a}}$

(3) $L_{\tilde{X}} d x^{h}=\partial_{m} v^{h} d x^{m}$

(4) $L_{\tilde{X}} \delta y^{h}=-\left\{y^{b} v^{c} R_{m c b}^{h}-v^{\bar{b}} \Gamma_{b m}^{h}-E_{m}\left(v^{\bar{h}}\right)\right\} d x^{m}$

$$
-\left\{v^{b} \Gamma_{b m}^{h}-E_{\bar{m}}\left(v^{\bar{h}}\right)\right\} \delta y^{m} .
$$




\section{Results}

If $g=g_{i j} d x^{i} d x^{j}$ is the expression of the Riemannian metric $g$, the CheegerGromoll metric $g_{C G}$ is expressed in the adapted local frame by

$$
g_{C G}=g_{i j} d x^{i} d x^{j}+h_{i j} \delta y^{i} \delta y^{j}
$$

where $h_{i j}$ is the function on $\pi^{-1}(U)$ defined by $h_{i j}=\frac{1}{1+r^{2}}\left(g_{i j}+y^{s} y^{t} g_{i s} g_{t j}\right)$. For shortness we set $G_{1}=g_{i j} d x^{i} d x^{j}$ and $G_{2}=h_{i j} \delta y^{i} \delta y^{j}$. Therefore the Cheeger-Gromoll metric $g_{C G}$ can be expressed as follows:

$$
g_{C G}=G_{1}+G_{2} .
$$

We shall first state the following Lemma which is needed later on.

3.1 Lemma. The Lie derivatives $L_{\tilde{X}} G_{1}$ and $L_{\tilde{X}} G_{2}$ with respect to the fibrepreserving vector field $\tilde{X}$ are given as follows:

$$
\begin{array}{ll}
\text { (1) } & L_{\tilde{X}} G_{1}=\left(L_{X} g_{i j}\right) d x^{i} d x^{j} \\
(2) & L_{\tilde{X}} G_{2}=-2 h_{m j}\left\{y^{b} v^{c} R_{i c b}^{m}-v^{\bar{b}} \Gamma_{b i}^{m}-E_{i}\left(v^{\bar{m}}\right)\right\} d x^{i} \delta y^{j} \\
& +\left\{L_{X} h_{i j}-2 h_{m j} \nabla_{i} v^{m}+2 h_{m j} E_{\bar{i}}\left(v^{\bar{m}}\right)\right. \\
& \left.+\frac{1}{1+r^{2}} v^{\bar{m}} y^{s}\left(-2 g_{m s} h_{i j}+g_{m j} g_{i s}+g_{s j} g_{i m}\right)\right\} \delta y^{i} \delta y^{j}
\end{array}
$$

where $L_{X} g_{i j}$ denote the components of the Lie derivative $L_{X} g$, and also $\nabla_{i} v^{m}$ denote the components of the covariant derivative of $X$.

Proof. Proof of this Lemma is similar to proof of the Proposition 2.3 of Yamauchi [31].

3.2 Theorem. Let $\left(T M, g_{C G}\right)$ be the tangent bundle with the Cheeger-Gromoll metric of a Riemannian manifold $(M, g)$. Let

(i) $X$ be an infinitesimal homothetic transformation on $(M, g)$, with $L_{X} g=$ $\Omega g$, for some constant $\Omega$;

(ii) $Y$ be a parallel vector field on $(M, g)$;

(iii) $A$ be a $(1,1)$-tensor field on $M$ which satisfies the followings

$$
\begin{aligned}
& \left(A_{1}\right) \quad g_{i k} A_{j}^{k}+g_{k j} A_{i}^{k}=2 \Omega g_{i j}, \\
& \left(A_{2}\right) \quad \nabla_{i} A_{j}^{k}+X^{l} R_{l i j}^{k}=0 .
\end{aligned}
$$

Then the vector field $\tilde{X}$ on $T M$ defined by

$$
\text { (\#) } \quad \tilde{X}={ }^{C} X+\gamma A+{ }^{V^{\prime}} Y
$$


is an infinitesimal fibre-preserving conformal transformation on $\left(T M, g_{C G}\right)$.

Conversely, every infinitesimal fibre-preserving conformal transformation on $\left(T M, g_{C G}\right)$ is of the form $(\sharp)$.

Let $T M$ be the tangent bundle over $M$ with the Cheeger-Gromoll metric $g_{C G}$, and let $\tilde{X}$ be an infinitesimal fibre-preserving conformal transformation on $\left(T M, g_{C G}\right)$ such that

$$
L_{\tilde{X}} g_{C G}=2 \Omega g_{C G} .
$$

By means of Lemma 3.1, we have

$$
\begin{aligned}
& \left(L_{X} g_{i j}\right) d x^{i} d x^{j}-2 h_{m j}\left\{y^{b} v^{c} R_{i c b}^{m}-v^{\bar{b}} \Gamma_{b i}^{m}-E_{i}\left(v^{\bar{m}}\right)\right\} d x^{i} \delta y^{j} \\
+ & {\left[L_{X} h_{i j}-2 h_{m j} \nabla_{i} v^{m}+2 h_{m j} E_{\bar{i}}\left(v^{\bar{m}}\right)\right.} \\
+ & \left.\frac{1}{1+r^{2}} v^{\bar{m}} y^{s}\left(-2 g_{m s} h_{i j}+g_{m j} g_{i s}+g_{s j} g_{i m}\right)\right] \delta y^{i} \delta y^{j} \\
= & 2 \Omega g_{i j} d x^{i} d x^{j}+2 \Omega h_{i j} \delta y^{i} \delta y^{j} .
\end{aligned}
$$

Comparing both sides of the above equation, we obtain the following three relations:

$$
\begin{gathered}
L_{X} g_{i j}=2 \Omega g_{i j} \\
y^{b} v^{c} R_{i c b}^{m}-v^{\bar{b}} \Gamma_{b i}^{m}-E_{i}\left(v^{\bar{m}}\right)=0 \\
L_{X} h_{i j}-2 h_{m j} \nabla_{i} v^{m}+2 h_{m j} E_{\bar{i}}\left(v^{\bar{m}}\right) \\
+\frac{1}{1+r^{2}} v^{\bar{m}} y^{s}\left(-2 g_{m s} h_{i j}+g_{m j} g_{i s}+g_{s j} g_{i m}\right)=2 \Omega h_{i j} .
\end{gathered}
$$

First all, we shall study the particular cases ${ }^{C} X, \gamma A,{ }^{\prime} Y$. Using (3.2)(3.4) and the local expressions of ${ }^{C} X, \gamma A, V^{\prime} Y$ with respect to the adapted frame, one easily proves, by direct computation, the following Lemmas.

3.3 Lemma. In order that a complete lift ${ }^{C} X$ to $T M$ of a vector field $X$ on $M$ be an infinitesimal fibre-preserving conformal transformation of $\left(T M, g_{C G}\right)$, it is necessary and sufficient that $X$ is an infinitesimal homothetic transformation of $(M, g)$.

3.4 Lemma. Let $A$ be a $(1,1)$-tensor field on $(M, g)$ satisfying the conditions $\left(A_{1}\right)$ and $\left(A_{2}\right)$ in Theorem 3.2. Then $\gamma A$ is an infinitesimal fibre-preserving conformal transformation on $\left(T M, g_{C G}\right)$.

3.5 Lemma. Let $Y$ be a vector field on $(M, g)$ which is parallel with respect to the Levi-Civita connection of $g$. Then ${ }^{V^{\prime}} Y$ is an infinitesimal fibre-preserving conformal transformation on $\left(T M, g_{C G}\right)$. 
Proof. Since sufficiency is shown by Lemma 3.3, Lemma 3.4 and Lemma 3.5, we now show necessity. We consider the 0 -section $\left(y^{i}=0\right)$ in the coordinate neighborhood $\pi^{-1}(U)$ in $T M$ and its neighborhood $W$. For a vector field $\tilde{X}=v^{i} E_{i}+v^{\bar{i}} E_{\bar{i}}$ on $T M$, and $(x, y)=\left(x^{i}, y^{i}\right)$ in $W$, we can write, by Taylor's theorem,

$$
\begin{aligned}
& v^{i}(x, y)=v^{i}(x, 0)+\left(\partial_{\bar{r}} v^{i}\right)(x, 0) y^{r}+\frac{1}{2}\left(\partial_{\bar{r}} \partial_{\bar{s}} v^{i}\right)(x, 0) y^{r} y^{s}+\ldots+[*]_{\lambda}^{i}, \\
& v^{\bar{i}}(x, y)=v^{\bar{i}}(x, 0)+\left(\partial_{\bar{r}} v^{\bar{i}}\right)(x, 0) y^{r}+\frac{1}{2}\left(\partial_{\bar{r}} \partial_{\bar{s}} v^{\bar{i}}\right)(x, 0) y^{r} y^{s}+\ldots+[*]_{\lambda}^{\bar{i}},
\end{aligned}
$$

where $[*]_{\lambda}^{I}(I=1,2, \ldots, 2 n)$ is of the form:

$$
[*]_{\lambda}^{I}=\frac{1}{\lambda !}\left(\partial^{\lambda} v^{I} / \partial y^{i_{1}} \partial y^{i_{2}} \ldots \partial y^{i_{\lambda}}\right)\left(x^{a}, \theta(x, y) y^{b}\right) y^{i_{1}} y^{i_{2}} \ldots y^{i_{\lambda}} ; 1 \leq i_{1}, \ldots, i_{\lambda} \leq n .
$$

The following lemma is valid.

3.6 Lemma. In the above situation, the following

$$
\begin{aligned}
& X=\left(X^{i}(x)\right)=\left(v^{i}(x, 0)\right), \\
& Y=\left(Y^{i}(x)\right)=\left(v^{\bar{i}}(x, 0)\right), \\
& K=\left(K_{r}^{i}(x)\right)=\left(\left(\partial_{\bar{r}} v^{i}\right)(x, 0)\right), \\
& E=\left(E_{r s}^{i}(x)\right)=\left(\left(\partial_{\bar{r}} \partial_{\bar{s}} v^{i}\right)(x, 0)\right), \\
& P=\left(P_{r}^{i}(x)\right)=\left(\left(\partial_{\bar{r}} v^{\bar{i}}\right)(x, 0)-\left(\partial_{r} v^{i}\right)(x, 0)\right)
\end{aligned}
$$

are tensor fields on $M$ [29].

For a fibre-preserving vector field $\tilde{X}=v^{i} E_{i}+v^{\bar{i}} E_{\bar{i}}$ on $T M$, with the notations of Lemma 3.6, we can write:

$$
\begin{gathered}
v^{i}(x, y)=X^{i} \\
v^{\bar{i}}(x, y)=Y^{i}+\tilde{P}_{r}^{i} y^{r}+\frac{1}{2} Q_{r s}^{i} y^{r} y^{s}+\ldots+[*]_{\lambda}^{\bar{i}},
\end{gathered}
$$

where $\tilde{P}_{r}^{i}$ and $Q_{r s}^{i}$ are given by $\tilde{P}_{r}^{i}=\left(\partial_{\bar{r}} v^{\bar{i}}\right)(x, 0)$ and $Q_{r s}^{i}=\left(\partial_{\bar{r}} \partial_{\bar{s}} v^{\bar{i}}\right)(x, 0)$.

Substituting (3.7) into (3.2), we have

$$
X^{m} \partial_{m} g_{i j}+\left(\partial_{i} X^{m}\right) g_{m j}+\left(\partial_{j} X^{m}\right) g_{i m}=2 \Omega g_{i j} .
$$

The equation (3.9) reduces to

$$
\nabla_{i} X_{j}+\nabla_{j} X_{i}=2 \Omega g_{i j} .
$$


Raising $j$ and contracting with $i$ in (3.10), it is easily seen that

$$
\Omega=\frac{1}{n}\left(\nabla_{i} X^{i}\right)
$$

i.e. the scalar function $\Omega$ on $T M$ depends only on the variables $\left(x^{i}\right)$ with respect to the induced coordinates $\left(x^{i}, y^{i}\right)$. Further, the vector field $X$ with the components $\left(X^{i}\right)$ is an infinitesimal conformal transformation on $M$. Since, by Lemma 3.3, ${ }^{C} X=X^{a} E_{a}+\left(y^{m} \nabla_{m} X^{a}\right) E_{\bar{a}}$ is an infinitesimal fibre-preserving conformal transformation on $\left(T M, g_{C G}\right), \tilde{X}-{ }^{C} X$ is also an infinitesimal fibrepreserving conformal transformation. Therefore, in the following, denoting $\tilde{X}-{ }^{C} X$ by the same letter $\tilde{X}$, one may assume that $X^{i}=0$ in (3.7). Then $\left(\tilde{P}_{i}^{r}\right)=\left(P_{i}^{r}\right)$ is a tensor field on $M$ by lemma 3.6.

Putting (3.7) and (3.8) into (3.3) [from now on, we omit this statement] and taking the part which does not contain $y^{r}$, we get

$$
\nabla_{i} Y^{m}=0
$$

Taking the part which does not contain $y^{r}$ in (3.4), we get

$$
g_{m j} \tilde{P}_{i}^{m}+g_{i m} \tilde{P}_{j}^{m}=2 \Omega g_{i j} .
$$

On differentiating $\partial_{\bar{k}}$ to the both sides of the equation (3.12), we obtain

$$
g_{i m} \partial_{\bar{k}} \tilde{P}_{\bar{j}}^{m}+g_{m j} \partial_{\bar{k}} \tilde{P}_{\bar{i}}^{m}=0 .
$$

Using (3.13) and the last equation in Lemma 2.3, we have

$$
\begin{gathered}
g_{i m} \partial_{\bar{k}} \partial_{\bar{j}}\left(v^{\bar{m}}\right)=-g_{m j} \partial_{\bar{k}} \partial_{\bar{i}}\left(v^{\bar{m}}\right) g_{m j}=-g_{m j} \partial_{\bar{i}} \partial_{\bar{k}}\left(v^{\bar{m}}\right) \\
=g_{m k} \partial_{\bar{i}} \partial_{\bar{j}}\left(v^{\bar{m}}\right)=g_{m k} \partial_{\bar{j}} \partial_{\bar{i}}\left(v^{\bar{m}}\right) \\
=-g_{m i} \partial_{\bar{j}} \partial_{\bar{k}}\left(v^{\bar{m}}\right)=-g_{m i} \partial_{\bar{k}} \partial_{\bar{j}}\left(v^{\bar{m}}\right),
\end{gathered}
$$

which gives

$$
\partial_{\bar{k}} \tilde{P}_{j}^{m}=0
$$

This shows that $\tilde{P}_{j}^{m}$ depends only on the variables $\left(x^{h}\right)$. Hence $\tilde{P}_{j}^{m}$ can be written as

$$
\tilde{P}_{j}^{m}=A_{j}^{m}
$$

where $A_{j}^{m}$ is a certain function which depends only on the variables $\left(x^{h}\right)$.

The coefficient of $y^{r}$ in $(3.3)$, by (3.14), gives

$$
X^{c} R_{i c r}^{m}-\nabla_{i} A_{r}^{m}=0
$$


or equivalently

$$
X^{c} R_{i c r l}-\nabla_{i} A_{r l}=0 .
$$

By (3.14), (3.12) is written as

$$
g_{m j} A_{i}^{m}+g_{i m} A_{j}^{m}=2 \Omega g_{i j}
$$

Applying the covariant derivative $\nabla_{k}$ to the both sides of the last equation, we obtain

$$
\nabla_{k}\left(A_{i j}\right)+\nabla_{k}\left(A_{j i}\right)=2\left(\nabla_{k} \Omega\right) g_{i j}
$$

Combining the last identity with (3.15), we get $\nabla_{k} \Omega=\partial_{k} \Omega=0$. This together with connectedness of $M$ shows that the scalar function $\Omega$ is constant.

Taking the coefficient of $y^{r}$ in (3.4), we get

$$
Y^{m}\left(-2 g_{m r} g_{i j}+g_{m j} g_{i r}+g_{r j} g_{i m}\right)+g_{m j} Q_{r i}^{m}+g_{m i} Q_{r j}^{m}=0 .
$$

We put $Q_{r s}^{i}=-2 Y^{i} g_{r s}+\left(Y^{k} g_{k r} \delta_{s}^{i}+Y^{k} g_{k s} \delta_{r}^{i}\right)+T_{r s}^{i}$. By a simple calculation, using (3.17), we can verify that $g_{m j} T_{i r}^{m}+g_{m i} T_{j r}^{m}=0$. If we put $T_{i r j}=g_{m j} T_{i r}^{m}$, then $T_{i r j}$ is symmetric in $i$ and $r$, and skew-symmetric $i$ and $j$. Hence $T_{i r j}=0$. That is

$$
Q_{r s}^{i}=-2 Y^{i} g_{r s}+\left(Y^{k} g_{k r} \delta_{s}^{i}+Y^{k} g_{k s} \delta_{r}^{i}\right) .
$$
(3.18)

Finally, we consider the coefficient of $y^{r} y^{s}$ in (3.3), we get by virtue of

$$
-2\left(\nabla_{i} Y^{m}\right) g_{r s}+\left(\nabla_{i} Y^{k}\right) g_{k r} \delta_{s}^{m}+\left(\nabla_{i} Y^{k}\right) g_{k s} \delta_{r}^{m}=0 .
$$

In view of (3.11), the last equation holds.

Now, by (3.15) and (3.16), we see that $\gamma A$ is an infinitesimal fibre-preserving conformal transformation on $\left(T M, g_{C G}\right)$ by Lemma 3.4. By (3.11) and Lemma $3.5,{ }^{\prime} Y$ is an infinitesimal fibre-preserving conformal transformation on $\left(T M, g_{C G}\right)$.

Summing up we find that $\tilde{X} \in \Im_{0}^{1}(T M)$ is an infinitesimal fibre-preserving conformal transformation with respect to the Chegeer-Gromoll metric iff

$$
\begin{gathered}
\tilde{X}=X^{i} E_{i}+\left(Y^{i}+\tilde{P}_{s}^{i} y^{s}+\frac{1}{2} Q_{s r}^{i} y^{s} y^{r}\right) E_{\bar{i}} \\
=X^{i} E_{i}+\left(Y^{i}+y^{s}\left(\nabla_{s} X^{i}+A_{s}^{i}\right)+\left(1-r^{2}\right) Y^{i}+g_{k s} Y^{k} y^{s} y^{i}\right) E_{\bar{i}} \\
={ }^{C} X+\gamma A+{ }^{\prime} Y
\end{gathered}
$$

for each local coordinate systems $\left(x^{i}\right), i=1, \ldots, n$ on $M$. This proves the assertion and the conditions (i), (ii) and (iii) are direct consequences of (3.9), (3.11), (3.15), (3.16) 
The result follows immediately from Theorem 3.2 and from its Proof.

3.7 Corollary. Every infinitesimal fibre-preserving conformal transformation on $\left(T M, g_{C G}\right)$ is homothetic and it induces an infinitesimal homothetic transformation. Consequently, it is of the form $(\sharp)$.

It is known that an infinitesimal homothetic transformation in a compact Riemannian manifold is a Killing vector field [32]. Theorem 3.2 and Corollary 3.7 deliver a simple and surprising result on compact manifolds:

3.8 Corollary. Let $(M, g)$ be a compact Riemannian manifold and TM be the tangent bundle of $M . \tilde{X} \in \Im_{0}^{1}(T M)$ is an infinitesimal fibre-preserving conformal transformation with respect to the Chegeer-Gromoll metric on TM iff $\tilde{X}$ is a Killing vector field with respect to the the Chegeer-Gromoll metric on $T M$.

\section{Acknowledgements}

The authors express their gratitude to two anonymous referees for their very helpful suggestions.

\section{References}

[1] M. T. K. Abbassi, Note on the classification theorems of g-natural metrics on the tangent bundle of a Riemannian manifold $(M, g)$, Comment. Math. Univ. Carolin., 45 (2004), no. 4, 591-596.

[2] M. T. K. Abbassi, M. Sarih, On some hereditary properties of Riemannian g-natural metrics on tangent bundles of Riemannian manifolds, Differential Geom. Appl., 22 (2005), no. 1, 19-47.

[3] M. T. K. Abbassi, M. Sarih, On natural metrics on tangent bundles of Riemannian manifolds, Arch. Math., 41 (2005), 71-92.

[4] M. T. K. Abbassi, M. Sarih, Killing vector fields on tangent bundles with Cheeger-Gromoll metric, Tsukuba J. Math., 27 (2003), 295-306.

[5] M. Benyounes, E. Loubeau, C.M. Wood, The geometry of generalised Cheeger-Gromoll metrics. Tokyo J. Math., 32 (2009), no. 2, 287-312.

[6] M. C. Calvo, G. R. Keilhauer, Tensor fields of type (0,2) on the tangent bundle of a Riemannian Manifold, Geom. Dedicata, 71 (1998), no.2, 209-219. 
[7] J. Cheeger, D. Gromoll, On the structure of complete manifolds of nonnegative curvature, Ann. of Math., 96 (1972), 413-443.

[8] P. Dombrowski, On the geometry of the tangent bundles, J. reine and angew. Math., 210 (1962), 73-88.

[9] A. Gezer, On infinitesimal conformal transformations of the tangent bundles with the synectic lift of a Riemannian metric, Proc. Indian Acad. Sci. Math. Sci., 119 (2009), no. 3, 345-350.

[10] S. Gudmundsson, E. Kappos, On the geometry of the tangent bundles, Expo. Math., 20 (2002), 1-41.

[11] I. Hasegawa, K. Yamauchi, Infinitesimal conformal transformations on tangent bundles with the lift metric I+II, Sci. Math. Jpn., 57 (2003), no.1, 129-137.

[12] I. Kolar, P. W. Michor, J. Slovak, Natural operations in differential geometry, Springer-Verlang, Berlin, 1993.

[13] O. Kowalski, M. Sekizawa, Natural transformation of Riemannian metrics on manifolds to metrics on tangent bundles-a classification, Bull. Tokyo Gakugei Univ., 40 (1988), no. 4, 1-29.

[14] M. I. Munteanu, Some aspects on the geometry of the tangent bundles and tangent sphere bundles of a Riemannian manifold, Mediterr. J. Math., 5 (2008), no.1, 43-59.

[15] E. Musso, F. Tricerri, Riemannian Metrics on Tangent Bundles, Ann. Mat. Pura. Appl., 150 (1988), no. 4, 1-19.

[16] V. Oproiu, Some classes of general natural almost Hermitian structures on tangent bundles, . Rev. Roumaine Math. Pures Appl., 48 (2003), no. 5-6, 521-533.

[17] V. Oproiu, A generalization of natural almost Hermitian structures on the tangent bundles, . Math. J. Toyama Univ., 22 (1999), 1-14.

[18] V. Oproiu, N. Papaghiuc, Some new geometric structures of natural lift type on the tangent bundle, Bull. Math. Soc. Sci. Math. Roumanie (N.S.), 52 (2009), no. 3, 333-346.

[19] V. Oproiu, N. Papaghiuc, General natural Einstein Kähler structures on tangent bundles, Differential Geom. Appl., 27 (2009), no. 3, 384392. 
[20] V. Oproiu, N. Papaghiuc, Classes of almost anti-Hermitian structures on the tangent bundle of a Riemannian manifold, An. Stiin. Univ. Al. I. Cuza Iasi. Mat. (N.S.), 50 (2004), no. 1, 175-190.

[21] V. Oproiu, N. Papaghiuc, On the geometry of tangent bundle of a (pseudo-) Riemannian manifold, An. tiin. Univ. Al. I. Cuza Iai. Mat. (N.S.), 44 (1998), no. 1, 67-83.

[22] N. Papaghiuc, Some locally symmetric anti-Hermitian structures on the tangent bundle, An. tiin?. Univ. Al. I. Cuza Iai. Mat. (N.S.), 52 (2006), no. 2, 277-287.

[23] N. Papaghiuc, Some geometric structures on the tangent bundle of a Riemannian manifold, Demonstratio Math., 37 (2004), no. 1, 215-228.

[24] N. Papaghiuc, A locally symmetric pseudo-Riemannian structure on the tangent bundle, Publ. Math. Debrecen, 59 (2001), no. 3-4, 303-315.

[25] N. Papaghiuc, A Ricci flat pseudo-Riemannian metric on the tangent bundle of a Riemannian manifold, Colloq. Math., 87 (2001), no. 2, 227-233.

[26] A. A. Salimov, S. Kazimova, Geodesics of the Cheeger-Gromoll metric, Turk. J. Math., 33 (2009), 99-105.

[27] S. Sasaki, On the differential geometry of tangent bundles of Riemannian manifolds, Tohoku Math. J., 10 (1958), 338-358.

[28] M. Sekizawa, Curvatures of tangent bundles with Cheeger-Gromoll metric, Tokyo J. Math., 14 (1991), 407-417.

[29] S. Tanno, Killing vectors and geodesic flow vectors on tangent bundles, J. Reine Angew. Math., 282 (1976), 162-171.

[30] K. Yamauchi, On infinitesimal conformal transformations of the tangent bundles with the metric I+III over Riemannian manifold, Ann Rep. Asahikawa. Med. Coll., 16 (1995), 1-6.

[31] K. Yamauchi, On infinitesimal conformal transformations of the tangent bundles over Riemannian manifolds, Ann Rep. Asahikawa. Med. Coll., 15 (1994), 1-10.

[32] K. Yano, On harmonic and Killing vector fields, Annals of Math., 55 (1952), 38-45. 
[33] K. Yano, S. Ishihara, Tangent and Cotangent Bundles, Marcel Dekker, Inc., New York 1973.

Aydin Gezer(Corresponding author),

Department of Mathematics,

Ataturk University, Faculty of Science,

25240, Erzurum Turkey. Email: agezer@atauni.edu.tr

Lokman Bilen,

Igdir University, Igdir Vocational School,

76000, Igdir-Turkey.

Email: lokman.bilen@igdir.edu.tr 
